\title{
Low-cost digital polariscope for dynamic photoelasticity
}

\author{
Anand K. Asundi, MEMBER SPIE \\ M. R. Sajan \\ University of Hong Kong \\ Department of Mechanical Engineering \\ Hong Kong \\ E-mail: H9190245@hkucc.hku.hk
}

\begin{abstract}
A low-cost dynamic polariscope is presented. The experimental arrangement is based on the delayed-microflash technique. A LED is used as the flash source. Dynamic photoelastic patterns are recorded after different time delays from the loading point using a CCD camera operating in the time-delay and integration mode. Unlike the conventional photographic techniques, the experiments need not be performed in total darkness. Digital recording provides instantaneous visualization of the patterns and rapid repeatability.
\end{abstract}

Subject terms: dynamic photoelasticity; high-speed photography; pulsed LED; time-delay and integration imaging; dynamic visualization.

Optical Engineering 33(9), 3052-3055 (September 1994).

\section{Introduction}

The photoelastic technique is a widely accepted tool for stress analysis on mechanical models. This noncontact method provides whole-field stress patterns which can be easily interpreted. Besides static stress analysis, photoelasticity is also applied in dynamic situations. Three recording systems are commonly used for registering the dynamic stress patterns in synchronization with the loading of the model. ${ }^{1}$ The first method relies on a high-speed rotation camera, which records the stress patterns successively on detecting the loading trigger. A Cranz-Schardin system is used in the second method. The third method utilizes intense flashes of very short duration, of the order of a few microseconds, to record the image on a high-speed film. Here only one fringe pattern is recorded at a preset time delay from loading, and the experiment needs to be repeated with different time delays to record all required fringe patterns. However, the fringe patterns recorded are larger in size with better resolution. This method is often used to enhance a particular frame obtained from either of the first two methods for detailed analysis.

Though static photoelastic setups are widely established in educational and research laboratories, dynamic techniques are not very popular. The main reason for this is the high capital cost of the equipment. In this paper, a low-cost dynamic photoelastic polariscope is described, in which the illumination is provided by a pulsed light-emitting diode (LED).

\section{Illumination and Imaging}

Low-modulus materials such as urethane rubber are commonly used for dynamic photomechanical experiments, as

Paper 17113 received Nov. 10, 1993; revised manuscript received Mar. 17, 1994; accepted for publication Mar. 18, 1994. (C) 1994 Society of Photo-Optical Instrumentation Engineers. 0091-3286/94/\$6.00. the stress wave velocity in these materials is ${ }^{2}$ about $200 \mathrm{~m} / \mathrm{s}$. The suggested microflash width for the urethane rubber in a previous dynamic photoelastic experiment is $20 \mu \mathrm{s}$ or less, ${ }^{3}$ which can freeze the slow-moving stress wave patterns. For high-modulus materials the stress wave velocity can be up to 10 times that of urethane rubber, and the fringes move considerably within the microflash time of $20 \mu \mathrm{s}$, causing smear in the recorded pattern. Therefore the pulse width should be reduced to about $1 \mu \mathrm{s}$. Since the duration of the flash in these experiments is very short, specially designed high-power flashes are necessary to generate sufficient light intensity to expose the photographic film.

A CCD camera is used in this experiment to record the fringe patterns. The CCD being more sensitive than the photographic film, it is possible to use less intense and lowerpower sources, such as LEDs, for the microflash. A LED emitting red, which is near the peak sensing wavelength $(700 \mathrm{~nm})$ of the camera, is used as the light source in the system. The LEDs operate at $5 \mathrm{~V}$, and so-called superbright models, costing about 50 U.S. cents, are available at local radio electronic shops.

The brightness of the LED depends on the current flowing through it. Typical current versus brightness characteristics of a LED are as depicted in Fig. 1. The brightness of the LED increases proportionally to the current, but there is a tradeoff between the intensity and the LED lifetime. Some of the tested LEDs are damaged at current levels in excess of $200 \mathrm{~mA}$. Hence the suggested operating current is $20 \mathrm{~mA}$, which provides sufficient illumination. However, when used in pulsed mode, it may be possible to use higher operating

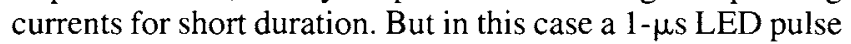
at $20 \mathrm{~mA}$ is adequate for recording a clear photoelastic pattern on a CCD camera. This suggests, and preliminary tests confirm, that the system could be used for dynamic investigations on stiffer photoelastic materials. 


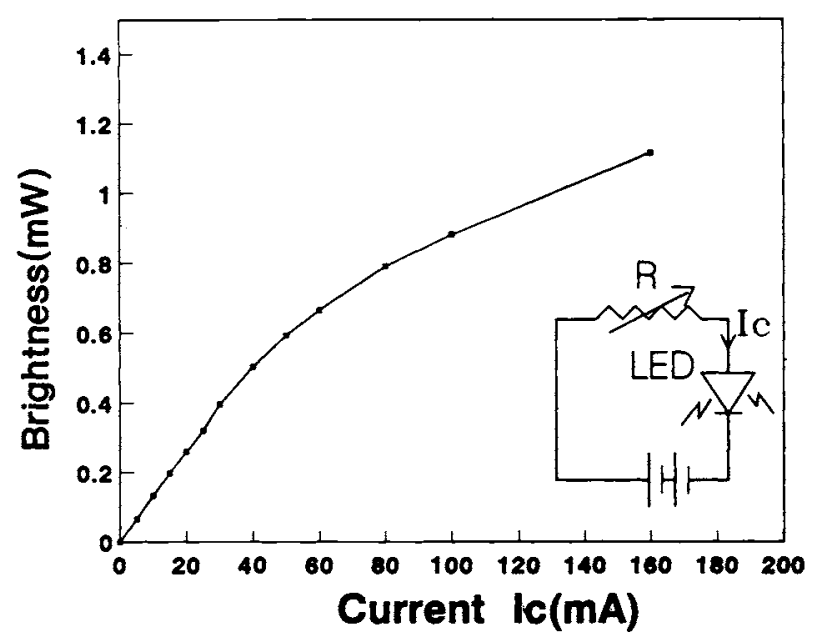

Fig. 1 Output power of the LED as a function of current.

The digital camera (EDC-1000, Electrim Corporation, Princeton, NJ) is capable of operating in the conventional full-frame imager mode as well as in the time delay and integration (TDI) mode. ${ }^{4}$ The TDI-mode performance is analogous to that of a drum camera for conventional film. Details of the camera operation are discussed elsewhere. ${ }^{5,6}$ Unlike the popular frame transfer mode, in the TDI mode the charge collection sites are shifted row by row at a user-specified speed. While shifting, the accumulated charges are integrated columnwise. Thus any one line in the TDI image represents the columnwise integrated charge value accumulated by all the rows in the CCD array. The advantage of using this mode of operation is that the camera is set into motion prior to the event that would be captured digitally at some stage during the recording. There is no lost film, and synchronization of the event with shuttering is avoided.

The camera is coupled to a PC486 through an interface card, making it possible to save the image in the computer memory. The size of a display frame is 192 columns $\times 165$ lines of pixels. The storage buffer has the capacity to save up to 3000 lines as specified by the user. Accordingly, the minimum and maximum size of the saved image are $192 \times 1$ pixels and $192 \times 3000$ pixels, respectively. The scanning speed (shifting speed of the charge collection sites) of the camera can be varied with function keys from 1 to 247,000 lines/min. Obviously, in the TDI image of a motionless scene with constant illumination, higher scanning speed will reduce the pixel intensity proportionately.

\section{Experiment and Results}

The bright-field polariscope arrangement used for recording the photoelastic patterns is depicted in Fig. 2. The photoelastic model used in the experiment is a $142-\mathrm{mm} \times 30$ $\mathrm{mm} \times 6-\mathrm{mm}$ bar of polyurethane rubber (PSM-4, Photoelastic Inc., Raileigh, NC) and is fixed in a groove carved in a wooden block. Dynamic loading is by a drop weight mechanism, provided by a ball of mass $27 \mathrm{~g}$ falling from a height of $1 \mathrm{~m}$. Due to the impact of the ball, stress waves are generated and propogate down the length of the bar. The builtin epoxy lens of the LED has a diameter of $5 \mathrm{~mm}$ and projects the light onto the collimating lens L1, thereby simplifying the optical alignment. The camera is fixed near the focal point

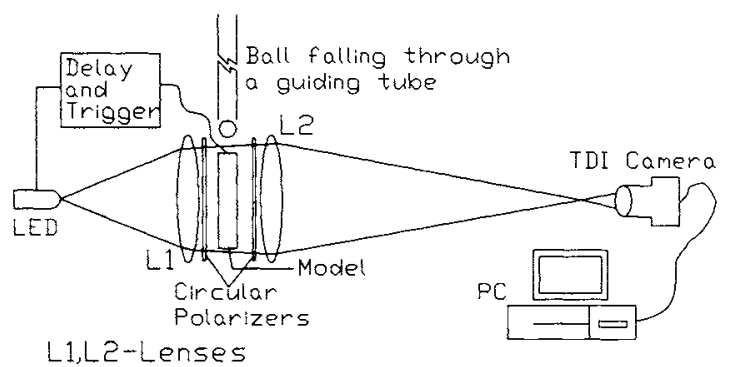

Fig. 2 Schematic of the experimental setup.

of lens L2 so as to get a clear image of the model under test. The alignments are first done with the camera operating in the standard full-frame imager mode and later switched to the TDI mode at a scanning speed of $20,000 \mathrm{lines} / \mathrm{min}$. The image-saving option of the camera is switched on just before releasing the ball. An aluminum foil contact attached to the top of the specimen detects the ball impact and triggers the delay circuit. After the pre-fixed time delay a $1-\mu$ s pulse is given to the LED through an amplifier. From the saved image, the photoelastic pattern is cut out using image-processing software. The experiment is repeated for larger time delays, and the resulting photoelastic patterns are compiled together for the dynamic visualization of stress wave propagation. Figure 3 shows the stress wave patterns recorded at intervals of $100 \mu \mathrm{s}$. The CCD array of the camera is of size $2.64 \mathrm{~mm} \times 2.64 \mathrm{~mm}$ and has a pixel resolution 192 (horizontal) $\times 165$ (vertical), which is much inferior to the currently available models. The resolution of the present imaging setup relative to the model dimension is $1 \mathrm{~mm}$ per pixel.

Initial investigations revealed the possibility of using the setup for dynamic analysis of high-modulus materials where the stress wave velocity is as much as $1800 \mathrm{~m} / \mathrm{s}$. A typical fringe pattern obtained from a polycarbonate (PSM-1, Photoelastic Inc., Raleigh, NC) specimen, recorded $80 \mu \mathrm{s}$ after impact using the same experimental configuration, is depicted in Fig. 4(a). A 1- $\mu$ s LED pulse is used as the microflash. After digital contrast enhancement, the image appears as in Fig. 4(b). The intensity variation near the central line along the length of the specimen is plotted in Fig. 5. The minimum and maximum intensity values correspond to dark and bright fringes, respectively. In the bright-field polariscope arrangement the bright fringes are assigned integer fringe order, and the dark fringes half-integer order. The fringes are numbered in the order of appearance. Accordingly the black fringe at the bottom of the bar is given the order 0.5 and represents the stress wave front. The fringe order, which is proportional to the shear stress, is plotted against the length of the bar in the same figure.

Currently, the authors are developing a Cranz-Schardintype multiple-LED camera which will give up to 16 dynamic patterns from the loading point at a pre-fixed time interval. This will be the subject of a future publication.

\section{Conclusion}

A low-cost dynamic photoelastic setup is presented. The LED provides a short-pulse alternative to microflash equipment. In addition to the cost reduction, the setup has the advantage of using a low-voltage circuit. Higher-current pulses can be employed to enhance the light intensity for recording patterns 
PSM-4 Bar. LED Flash(1 $\mu \mathrm{s})$. Time after impact in $\mu \mathrm{s}$
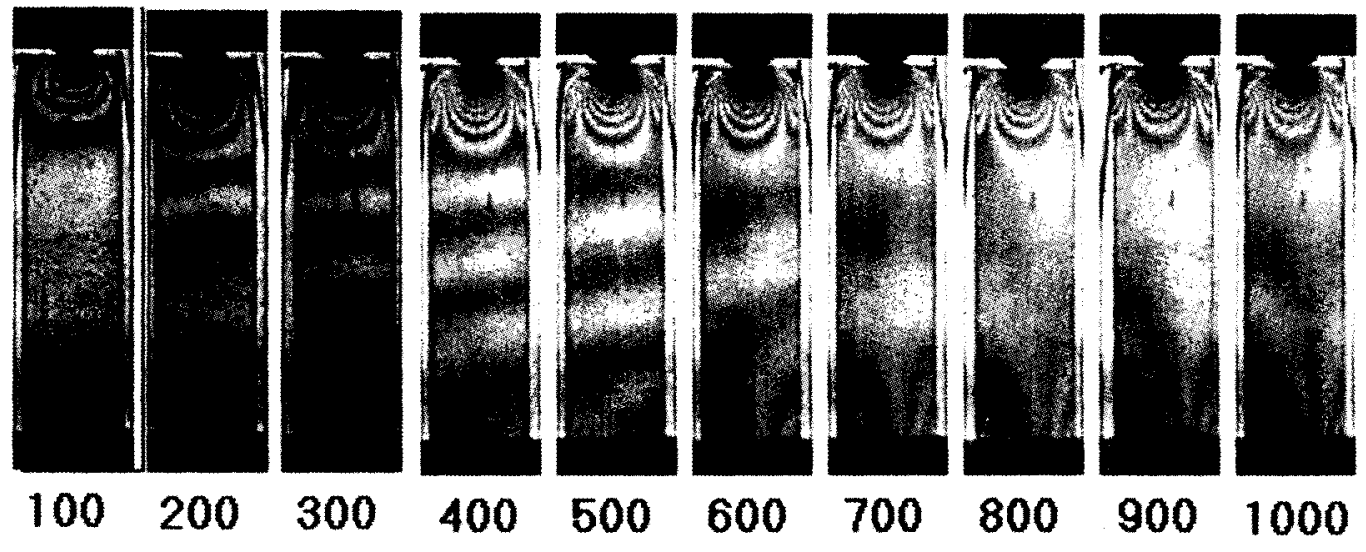

300

$400 \quad 500$

600

700800

9001000

Fig. 3 Dynamic photoelastic patterns from a polyurethane bar.

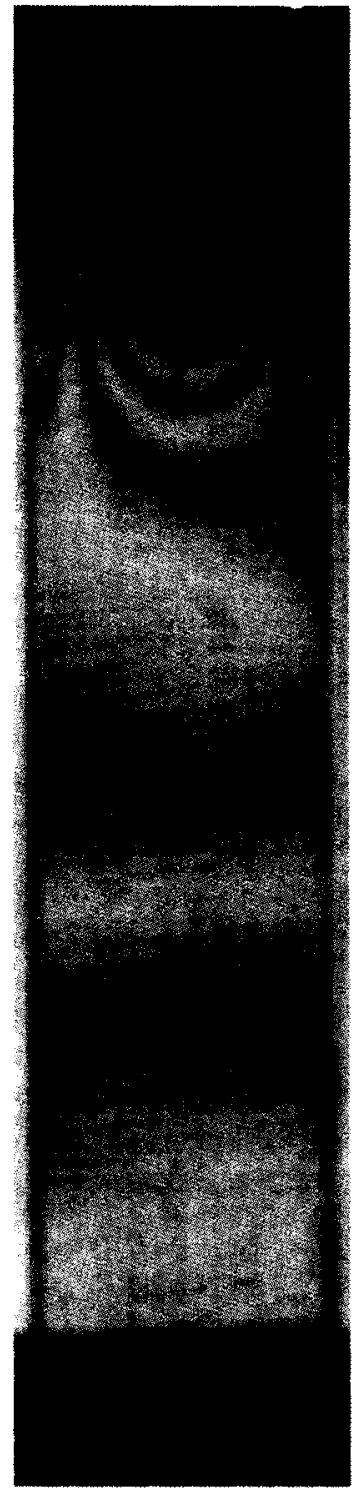

(a)

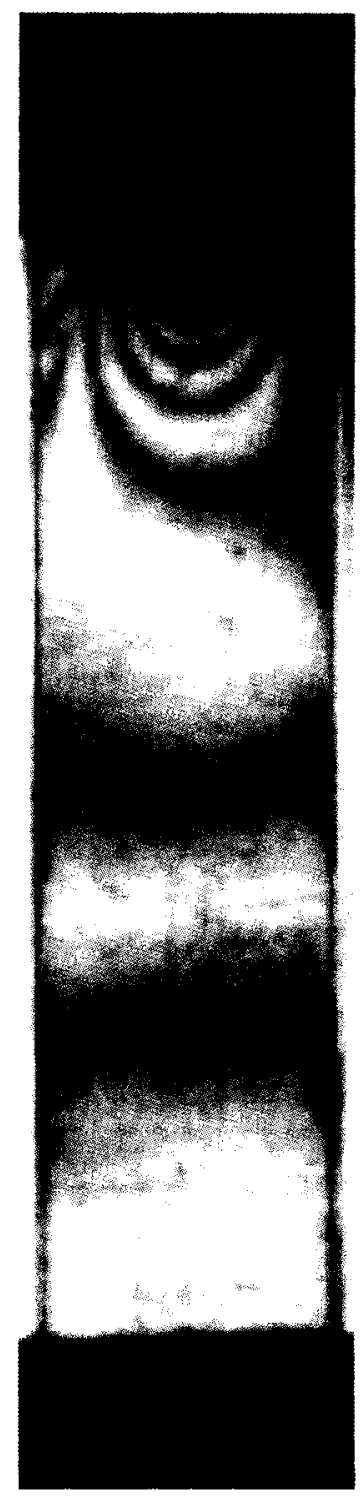

(b)

Fig. 4 (a) Photoelastic pattern recorded from a polycarbonate bar $80 \mu s$ after the ball impact. (b) After contrast enhancement.

from large photoelastic models. The experiments need not be performed in complete darkness. The digital recording procedure reduces the tedium involved in film processing and provides rapid repeatability.

\section{Acknowledgments}

We thankfully acknowledge assistance from the University Research Council and the Committee for Research and Conference Grants, The University of Hong Kong.

\section{References}

1. J. W. Dally, "An introduction to dynamic photoelasticity," Exp. Mech. 20, 409-416 (1980)

2. J. A. Clark and A. J. Durelli, "An introduction to dynamic photoelasticity (discussion)," Exp. Mech. 23, 42-48 (1983).

3. A. J. Durelli and W. F. Riley, Introduction to Photomechanics, pp. 123-135, Prentice-Hall, New York (1965).

4. D. L. Gilblom, "TDI imaging in industrial inspection," Proc. SPIE 1194, 36-45 (1989).

5. A. Asundi and M. R. Sajan, "Distortion and defect identification on curved objects," Proc. SPIE 1907, 218-223 (1993).

6. A. Asundi and M. R. Sajan, "Digital dynamic photoelasticity," Opt. Lasers Eng. 20, 135-140 (1994).

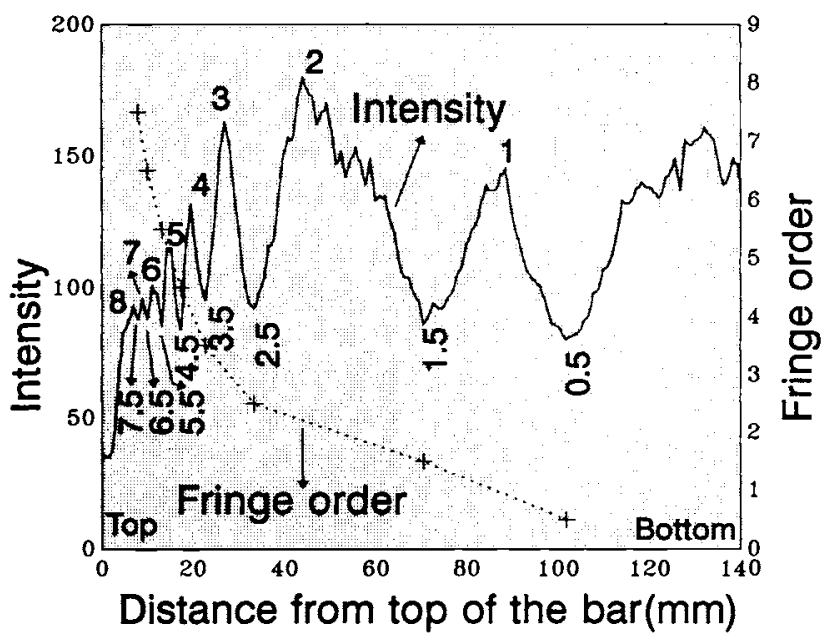

Fig. 5 Intensity variation and fringe distribution near the central line along the length of the bar. 


\section{LOW-COST DIGITAL POLARISCOPE FOR DYNAMIC PHOTOELASTICITY}

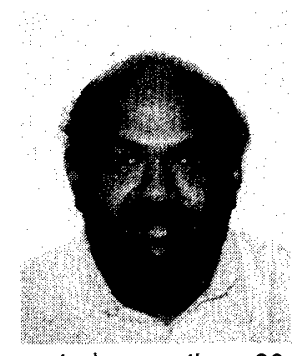

Anand K. Asundi (CEng) graduated from IIT Bombay and subsequently received his PhD from the State University of New York at Stony Brook. Following a brief tenure as research associate at Virginia Tech., he joined the University of Hong Kong, where he is currently a reader. His research interests are in experimental mechanics, image processing and machine vision, composite materials, and fracture and damage mechanics. He has published and presented more than 80 papers in these areas. He is a member of SPIE, OSA, SEM, ASME, and IMechE.

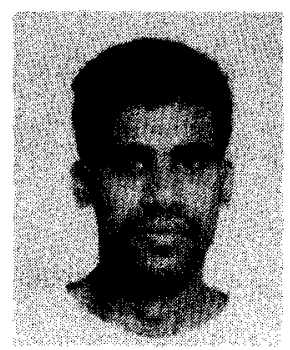

M. R. Sajan received his MS in optical instrumentation from the Indian Institute of Science in 1991. Currently he is pursuing a PhD in the Department of Mechanical Engineering, The University of Hong Kong. $\mathrm{He}$ is interested in optical interferometry, dynamic visualization, photomechanics, and automated visual inspection. 